\title{
Correlações Genéticas entre Características Produtivas de Fêmeas em um Rebanho da Raça Canchim ${ }^{1}$
}

\author{
Fernando Molinari Talhari² ${ }^{2}$ Maurício Mello de Alencar ${ }^{3}$, Arthur dos Santos Mascioli ${ }^{4}$, Ana Mary \\ da Silva ${ }^{5}$, Pedro Franklin Barbosa ${ }^{6}$
}

\begin{abstract}
RESUMO - O objetivo deste trabalho foi estimar as correlações genéticas dos pesos à desmama (PD), aos 12 (P12) e aos 18 (P18) meses de idade com a idade (IPP) e o peso (PPP) ao primeiro parto, o peso adulto (PAD) e os parâmetros A (peso assintótico) e k (taxa de maturação) da curva de Von Bertalanffy de fêmeas, em um rebanho Canchim. Utilizou-se o método da máxima verossimilhança restrita, em análises bicaráter, com modelos que incluíram os efeitos fixos de grupo de contemporâneos e o efeito aleatório genético aditivo direto. Para PD, o modelo incluiu também a covariável idade da vaca ao parto (efeitos linear e quadrático) e os efeitos aleatórios genético aditivo materno e de ambiente permanente. Para PAD, o modelo incluiu também os efeitos fixos de idade da vaca. As médias das estimativas de herdabilidade foram iguais a: 0,37 (PD); 0,31 (P12); 0,35 (P18); 0,39 (A); 0,29 (k); 0,13 (IPP); 0,39 (PPP); e 0,42 (PAD). As correlações genéticas de PD com as outras características foram iguais a: 0,46 (parâmetro A); 0,02 (parâmetro k); -0,12 (IPP); 0,66 (PPP); e 0,42 (PAD). Com P12 as correlações genéticas foram iguais a 0,39 (parâmetro A); 0,31 (parâmetro k); -0,32 (IPP); 0,77 (PPP); e 0,66 (PAD) e com P18 foram iguais a 0,21 (parâmetro A); 0,42 (parâmetro k); -0,29 (IPP); 0,65 (PPP); e 0,60 (PAD). Estes resultados indicam que PD, P12, P18, PPP, PAD e os parâmetros A e $\mathrm{k}$ das fêmeas possuem variação genética aditiva suficiente para responderem à seleção massal e que a seleção para PD, P12 e P18 deve resultar em respostas correlacionadas desejáveis em IPP e k, mas indesejáveis em PPP e PAD.
\end{abstract}

Palavras-chave: bovinos de corte, curva de crescimento, idade ao primeiro parto, peso adulto, seleção

\section{Genetic Correlations among Reproductive and Growth Traits of Females, in a Canchim Cattle Herd}

ABSTRACT - The objective of this study was to estimate the genetic correlations of female body weights at weaning (BWW), 12 (W12) and 18 (W18) months of age with age at first calving (AFC), body weight at first calving (WFC), adult body weight (ABW), and the parameters for mature weight (A) and maturation rate (k) obtained using the Von Bertalanffy model, in a Canchim herd. The restricted maximum likelihood method, with two-trait analyses, was used with models that included the fixed effects of contemporary group and the random additive direct effect. For BWW the model also included the covariate age of cow at calving (linear and quadratic effects) and the additive maternal and the permanent environmental random effects, and for ABW the model included also the fixed effect of age of cow. The means of the heritability estimates were equal to: 0.37 (BWW), 0.31 (W12), 0.35 (W18), 0.39 (A), 0.29 (k), 0.13 (AFC), 0.39 (WFC), and $0.42(\mathrm{ABW})$. The genetic correlations between BWW and the other traits were: $0.46(\mathrm{~A}), 0.02(\mathrm{k}),-0.12(\mathrm{AFC}), 0.66$ (WFC) and $0.42(\mathrm{ABW})$. With W12 they were equal to $0.39(\mathrm{~A}), 0.31(\mathrm{k}),-0.32(\mathrm{AFC}), 0.77$ (WFC) and 0.66 (ABW), while with W18 they were equal to $0.21(\mathrm{~A}), 0.42(\mathrm{k}),-0.29(\mathrm{AFC}), 0.65$ (WFC) and 0.60 (ABW) These results indicate that selection to increase BWW, $\mathrm{W} 12, \mathrm{~W} 18, \mathrm{WFC}, \mathrm{ABW}$, and parameters A and $\mathrm{k}$ should result in direct genetic responses, and that selection to increase female body weights from weaning to 18 months of age should result in favorable responses in AFC and parameter k, but in unfavorable correlated responses in WFC and $\mathrm{ABW}$.

Key Words: adult body weight, age at first calving, beef cattle, growth curve parameters, selection

\section{Introdução}

Os pesos à desmama e ao sobreano são caracteríticas normalmente incluídas nos programas de avaliação genética de várias raças bovinas de corte no Brasil, em virtude de suas herdabilidades serem de magnitude média e de serem importantes na determinação da eficiência produtiva dos rebanhos. Entretanto, alguns autores têm observado correlação genética desfavorável entre peso em várias idades e

\footnotetext{
1 Trabalho realizado mediante contrato de cooperação entre a Embrapa e a UFSCar.

2 Estudante de pós-graduação da UFSCar, São Carlos, SP. Bolsista da FAPESP. E.mail: ftalhari@terra.com.br

3 Pesquisador da Embrapa Pecuária Sudeste, Caixa Postal 339, CEP: 13560-970, São Carlos, SP. Bolsista do CNPq.

E.mail: mauricio@cppse.embrapa.br

4 Professor de Melhoramento Genético Animal. E.mail: amascioli@netsite.com.br

5 Professora Assistente 1, Departamento de Zootecnia da UFRPE, Recife, PE. E.mail: aninha123@yahoo.com

6 Pesquisador da Embrapa Pecuária Sudeste. E.mail: pedro@cppse.embrapa.br
} 
características produtivas e de eficiência reprodutiva em bovinos de corte (Mariante, 1978; DeNise et al., 1983; Barbosa, 1991). Outros autores (Jenkins et al., 1991; Bullock et al., 1993; Silva et al., 2000) verificaram correlação genética positiva entre pesos em idades jovens e os pesos adulto e à maturidade em fêmeas, indicando que a seleção para maior peso em idades jovens deve aumentar o peso adulto das fêmeas. Antes de se investir em um programa de seleção para características de peso, é necessário que se conheçam as relações entre elas e as características de eficiência reprodutiva, de precocidade e de tamanho das fêmeas, dada a importância econômica das primeiras e o custo de manutenção do rebanho de vacas. O objetivo deste trabalho foi estimar as correlações genéticas dos pesos à desmama, aos $12 \mathrm{e}$ aos 18 meses de idade com o peso e a idade ao primeiro parto, o peso adulto e os parâmetros da curva de crescimento, em fêmeas de um rebanho da raça Canchim.

\section{Material e Métodos}

Os dados utilizados neste estudo são provenientes de animais do rebanho de bovinos da raça Canchim pertencente à Embrapa Pecuária Sudeste (CPPSE), localizada no município de São Carlos, região central do Estado de São Paulo. Os animais desse rebanho foram criados em regime exclusivo de pastagens com suplementação mineral durante o ano todo.

O manejo reprodutivo das fêmeas incluiu diferentes critérios de entrada em reprodução durante toda a existência do rebanho. De 1953 até 1975, as novilhas entravam em reprodução próximas aos 34 meses de idade e $360 \mathrm{~kg}$ de peso vivo. A partir de 1976, a idade foi reduzida para próximo dos 26 meses e cerca de $300 \mathrm{~kg}$ de peso e, a partir de 1979, a seleção de novilhas para entrada em reprodução foi feita por volta de 22 meses de idade, levando-se em consideração, além da idade, o seu peso e características raciais, procurando-se manter no rebanho apenas as novilhas que emprenharam na primeira estação de monta. Após o primeiro parto as vacas eram colocadas com touros após a desmama do bezerro. A partir de 1976 (exceto 1977), todas as vacas foram colocadas com touros durante a estação de monta, com exceção das prenhes. Os lotes de monta eram compostos por um touro e cerca de 30 vacas. A partir de 1979, começou-se a utilizar também a inseminação artificial. Em vários anos foram utilizadas duas estações de monta, uma no primeiro semestre e outra no segundo semestre, mas não tendo mês fixo para iniciar nem para terminar, tendo duração variável (dois a quatro meses). O descarte de vacas do rebanho se dava principalmente por motivos de doenças e, ou, acidente; a partir de 1977, iniciou-se o descarte de vacas consideradas de baixa fertilidade, ou seja, que não emprenhassem em duas estações de monta seguidas.

O rebanho em questão se mantém fechado desde sua formação, sendo que no período estudado os acasalamentos endogâmicos foram evitados e todos os cuidados sanitários necessários foram tomados. Mais informações sobre os outros tipos de manejo deste rebanho podem ser obtidos em Alencar et al. (1981) e Barbosa (1991).

Neste trabalho foram estimadas as herdabilidades e as correlações genéticas dos pesos à desmama (PD), aos 12 (P12) e aos 18 (P18) meses de idade, da idade (IPP) e do peso (PPP) ao primeiro parto, do peso adulto (PAD) e dos parâmetros $\mathrm{A}$ e $\mathrm{k}$ da curva de crescimento das fêmeas. Para este trabalho consideraram-se apenas os dados de desempenho dos animais criados em regime exclusivo de pastagem. Os dados dos animais que, por algum motivo, tiveram alimentação complementar foram descartados, considerando-se apenas as suas identificações e os seus pedigrees, com o objetivo de construir a matriz de parentesco.

Para PD, P12 e P18, foram utilizados os dados de 2804, 2613 e 2295 fêmeas nascidas de 1953 a 1996, respectivamente. PD, P12 e P18 foram padronizados para 240,365 e 550 dias, considerando-se os ganhos médio diários do nascimento à desmama, da desmama ao ano e do ano ao sobreano, respectivamente.

Para descrever o crescimento do animal em função do tempo, foi utilizado o modelo não-linear de Von Bertalanffy (Bertalanffy, 1957), $\mathrm{y}_{\mathrm{t}}=\mathrm{A}\left(1-\mathrm{Be}^{-\mathrm{kt}}\right)^{3}$ $+e_{t}$, que, segundo Freitas et al. (1998), descreve de forma satisfatória o crescimento das fêmeas do rebanho estudado. No modelo, o parâmetro A é o peso assintótico que representa o peso à maturidade, ou seja, o peso quando o tempo $t$ tende ao infinito, e o parâmetro $\mathrm{k}$ é a taxa de maturação, ou seja, a velocidade com que o animal atinge o peso assintótico. Para as estimativas dos parâmetros da curva de crescimento de Von Bertalanffy utilizaram-se o procedimento NLIN (SAS, 1996) e dados de 486 vacas nascidas de 1953 a 1975.

$O$ peso adulto (PAD) das fêmeas foi considerado como o peso logo após o parto, para vacas de 4, 5, 6

R. Bras. Zootec., v.32, n.4, p.880-886, 2003 
a 8 e $\geq 9$ anos de idade. Foi considerado apenas um peso para cada vaca e, no caso de a vaca possuir mais de um peso, utilizou-se o peso mais próximo de seis anos, idade em que o animal, provavelmente, já parou de crescer. Foram consideradas 956 vacas paridas de 1977 a 1997.

Para IPP, utilizaram-se os dados de 1466 fêmeas nascidas de 1953 a 1994. Para PPP foram considerados os dados de 927 fêmeas paridas de 1977 a 1997.

Para obtenção dos parâmetros genéticos, os componentes de variância e de covariância foram estimados pelo método da máxima verossimilhança restrita livre de derivadas (DFREML), utilizando-se o programa MTDFREML (Boldman et al., 1993), realizando-se análises de duas características de cada vez. O modelo matemático utilizado incluiu os efeitos fixos de grupo de contemporâneos (GC) e aleatórios genético aditivo direto. Utilizou-se o mesmo modelo matemático para todas as características, exceto para PD, cujo modelo incluiu também os efeitos aleatórios genético aditivo materno e de ambiente permanente, além da covariável idade da vaca ao parto (efeitos linear e quadrático) e para PAD, cujo modelo incluiu também os efeitos fixos de idade da vaca $(4,5,6$ a 8 e $\geq 9$ anos de idade). Para PD, P12, P18, IPP e os parâmetros A e k, os GC foram compostos pelo ano e pela época $(1=$ janeiro a março; 2 = abril a junho; 3 = julho a setembro; $4=$ outubro a dezembro) de nascimento. Para PPP e PAD, os GC foram compostos pelo ano e pela época do parto. Foram considera- dos GC com no mínimo cinco observações.

$\mathrm{Na}$ matriz de parentesco, os animais (6928) base utilizados foram os 5/8 Charolês + 3/8 Zebu que, acasalados entre si, produziram os primeiros bimestiços, denominados de Canchim.

\section{Resultados e Discussão}

As médias observadas e seus respectivos desvios-padrão foram iguais a: 195,2 e 33,8 kg; 205,2 e $38,9 \mathrm{~kg} ; 265,2$ e $45,6 \mathrm{~kg} ; 517,2$ e $119,5 \mathrm{~kg} ; 0,056$ e 0,017 mês $^{-1} ; 1305,3$ e 219,1 dias; 429,0 e $57,6 \mathrm{~kg}$; e 496,0 e 70,9 kg, para as características PD, P12, P18, A, k, IPP, PPP e PAD, respectivamente.

Nas Tabelas 1, 2 e 3 são apresentados os componentes de variância e covariância para PD, P12 e P18 e as demais características das fêmeas, respectivamente, obtidos por análise bicaráter. As estimativas de herdabilidade direta para PD variaram de 0,36 a 0,39 , apresentando média de 0,37 , enquanto as estimativas de herdabilidade materna variaram de 0,11 a 0,15 , com média de 0,13. Alencar et al. (1998), usando a mesma metodologia deste estudo e dados do mesmo rebanho, estimaram os valores de 0,29 e 0,10 para as herdabilidades direta e materna de PD. Mello et al. (2002) obtiveram, para o mesmo rebanho, as estimativas de 0,48 e 0,04 para as herdabilidades direta e materna de PD, utilizando o mesmo modelo, mas por análise tricaráter.

As estimativas de herdabilidade direta obtidas

Tabela 1 - Componentes $^{2}$ de (co)variância de PD (característica 1) e A, k, IPP, PPP e PAD (característica 2) de fêmeas da raça Canchim

Table 1 - Variance and covariance components ${ }^{2}$ of BWW (trait 1) and A, k, AFC, WFC and ABW (trait 2) of females of the Canchim breed

\begin{tabular}{|c|c|c|c|c|c|c|c|c|c|c|}
\hline \multirow[t]{2}{*}{$\begin{array}{l}\text { Caract. } 2^{1} \\
\text { Trait } 2^{1}\end{array}$} & \multicolumn{4}{|c|}{$\begin{array}{l}\mathrm{PD}(\text { caract. } 1)^{1} \\
B W W(\text { trait } 1)^{1}\end{array}$} & \multicolumn{3}{|c|}{$\begin{array}{l}\text { Caract. } 2^{*} \\
\text { Trait } 2^{*}\end{array}$} & \multicolumn{3}{|c|}{$\begin{array}{l}\text { Caract. } 1,2^{* *} \\
\quad \text { Trait } 1,2^{* *}\end{array}$} \\
\hline & $\sigma^{2}{ }_{a 1}$ & $\sigma_{\mathrm{m} 1}^{2}$ & $\sigma_{\text {per }}^{2}$ & $\sigma^{2}{ }_{\mathrm{e} 1}$ & $\sigma_{\mathrm{a} 1 \mathrm{~m} 1}$ & $\sigma^{2}{ }_{2}$ & $\sigma_{\mathrm{e} 2}^{2}$ & $\sigma_{\mathrm{a} 1 \mathrm{a} 2}$ & $\sigma_{\mathrm{e} 1 \mathrm{e} 2}$ & $\sigma_{\mathrm{a} 2 \mathrm{~m} 1}$ \\
\hline$\overline{\mathrm{A} / A}$ & 284 & 94 & 106 & 372 & -84 & 3846 & 5746 & 483 & -146 & -115 \\
\hline $\mathrm{K} / K$ & 297 & 88 & 112 & 364 & -90 & 6 & 12 & 230 & 380 & 150 \\
\hline $\mathrm{IPP} / A F C$ & 298 & 88 & 109 & 365 & -88 & 3560 & 26291 & -124 & -403 & -35 \\
\hline $\mathrm{PPP} / W F C$ & 283 & 101 & 97 & 372 & -78 & 1083 & 1592 & 363 & 73 & 22 \\
\hline $\mathrm{PAD} / A B W$ & 281 & 111 & 93 & 373 & -84 & 1440 & 1578 & 265 & 231 & 123 \\
\hline
\end{tabular}

${ }_{1}$ PD, A, k, IPP, PPP e PAD = peso à desmama $(\mathrm{kg})$, estimativas dos parâmetros A $(\mathrm{kg})$ e k (mês ${ }^{-1}$ ) da curva de crescimento, idade (dias) e peso $(\mathrm{kg})$ ao primeiro parto e peso $(\mathrm{kg})$ à idade adulta, respectivamente.

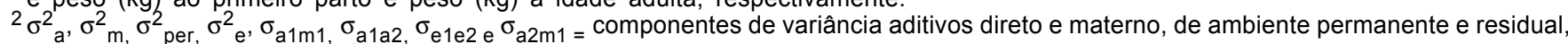
e de covariáncia entre os efeitos aditivos direto e materno, aditivos diretos, residuais e aditivos direto e materno, respectivamente.

* variâncias de k x 100.000; ${ }^{\star *}$ covariâncias com k x 10.000 .

1 'BWW, $A, k, A F C, W F C$ and $A B W=$ weaning weight $(\mathrm{kg})$, estimates of the parameters $A(\mathrm{~kg})$ and $\mathrm{k}\left(\mathrm{month}^{-1}\right)$ of the growth curve, age (days) and weight $(\mathrm{kg})$ at first calving, and adult weight $(\mathrm{kg})$, respectively.

${ }^{2} \sigma_{a}^{2}, \sigma_{m,}^{2} \sigma_{p e r}^{2} \sigma_{e}^{2}, \sigma_{a 1 m 1}, \sigma_{a 1 a 2}, \sigma_{e 1 e 2 \text { and }} \sigma_{m 1 a 2}=$ additive direct, additive maternal, permanent environmental and residual variance components, and additive - maternal, additive, residual and additive - maternal covariance components, respectively.

* variances of $k \times 100.000 ;{ }^{* *}$ covariances with $k \times 10.000$

\section{R. Bras. Zootec., v.32, n.4, p.880-886, 2003}


Tabela 2 - Componentes ${ }^{2}$ de (co)variância de P12 (característica 1) e A, k, IPP, PPP e PAD (característica. 2) de fêmeas da raça Canchim

Table 2 - Variance and covariance components ${ }^{2}$ of BW12 (trait 1) and A, k, AFC, WFC and ABW (trait 2) of females of the Canchim breed

\begin{tabular}{|c|c|c|c|c|c|c|}
\hline \multirow[t]{2}{*}{$\begin{array}{l}\text { Caract. } 2^{1} \\
\text { Trait } 2^{1}\end{array}$} & \multicolumn{2}{|c|}{$\begin{array}{c}\mathrm{P} 12(\text { caract. } 1)^{1} \\
\text { BW12 }(\text { trait } 1)^{1}\end{array}$} & \multicolumn{2}{|c|}{$\begin{array}{l}\text { Caract. } 2^{*} \\
\text { Trait } 2^{*} \\
\end{array}$} & \multicolumn{2}{|c|}{$\begin{array}{c}\text { Caract. } 1,2^{* *} \\
\text { Trait } 1,2^{* *} \\
\end{array}$} \\
\hline & $\sigma^{2}{ }^{2} 1$ & $\sigma_{\mathrm{e} 1}^{2}$ & $\sigma^{2}{ }_{a 2}$ & $\sigma_{\mathrm{e} 2}^{2}$ & $\sigma_{\mathrm{a} 1 \mathrm{a} 2}$ & $\sigma_{\mathrm{e} 1 \mathrm{e} 2}$ \\
\hline $\mathrm{A} / \mathrm{A}$ & 259 & 591 & 3510 & 6034 & 370 & -525 \\
\hline $\mathrm{K} / \mathrm{k}$ & 267 & 587 & 6 & 13 & 38 & 15 \\
\hline IPP/AFC & 266 & 587 & 3859 & 26514 & -325 & -693 \\
\hline PPP/WFC & 274 & 583 & 1127 & 1591 & 430 & 139 \\
\hline $\mathrm{PAD} / \mathrm{ABW}$ & 273 & 583 & 1169 & 1786 & 375 & 295 \\
\hline
\end{tabular}

1 P12, A, k, IPP, PPP e PAD = peso $(\mathrm{kg})$ aos 12 meses de idade, estimativas dos parâmetros A $(\mathrm{kg})$ e $\mathrm{k}\left(\mathrm{mês}^{-1}\right)$ da curva de crescimento, idade (dias) e peso $(\mathrm{kg})$ ao primeiro parto e peso $(\mathrm{kg})$ à idade adulta, respectivamente.

$2 \sigma_{a}^{2}, \sigma_{e}^{2}, \sigma_{\mathrm{a} 1 \mathrm{a} 2}$ e $\sigma_{\mathrm{e} 1 \mathrm{e} 2}=$ componentes de variância genético aditivo direto e residual e de covariância aditiva direta e residual, respectivamente.

* variâncias de k x 100.000; ${ }^{* *}$ covariâncias com k x 10.000

${ }^{1} B W 12, A, k, A F C, W F C$ and $A B W=$ body weight $(\mathrm{kg})$ at 12 months of age, estimates of the parameters $A(\mathrm{~kg})$ and $k\left(\mathrm{month}^{-1}\right)$ of the growth curve, age (days) and weight ( $\mathrm{kg}$ ) at first calving, and adult weight ( $\mathrm{kg})$, respectively.

${ }^{2} \sigma^{2}{ }_{a}, \sigma^{2}{ }_{e}, \sigma_{a 122}$ and $\sigma_{e 1 e 2}=$ genetic additive direct and residual variance components, and additive and residual covariance components.

* variances of $k \times 100.000 ;{ }^{*}$ covariances with $k \times 10.000$.

para P12 variaram de 0,31 a 0,32 , com média de 0,31 , concordando com os valores de 0,29 e 0,30 obtidos por Alencar et al. (1998) e Silva et al. (2000), mas inferiores ao de 0,63 encontrado por Mello et al. (2002). Para P18, as estimativas de herdabilidade direta variaram de 0,35 a 0,36 , com média de 0,35 , valores esses ligeiramente inferiores ao valor de 0,40 obtido por Silva (1999).

As herdabilidades diretas das demais características, A, k, IPP, PPP e PAD variaram de 0,37 a 0,40 ; 0,24 a 0,$34 ; 0,12$ a 0,$13 ; 0,37$ a 0,42 e 0,38 a 0,48 , apresentando médias iguais a 0,$39 ; 0,29 ; 0,13 ; 0,39$ e 0,42 , respectivamente. Esses valores concordam com aqueles obtidos por Silva et al. (2000) para o mesmo conjunto de dados, mas por análises unicaráter e bicaráter com P12 de machos.

As estimativas de herdabilidade obtidas neste trabalho indicam que a seleção para as características ligadas ao crescimento (PD, P12, P18, A, k, PPP e PAD) das fêmeas da raça Canchim do rebanho estudado, deve resultar em progresso genético; contudo, a estimativa de herdabilidade de IPP mostra que

Tabela 3 - Componentes $^{2}$ de (co)variância de P18 (característica 1) e A, k, IPP, PPP e PAD (característica 2) de fêmeas da raça Canchim

Table 3 - Variance and covariance components ${ }^{2}$ of BW18 (trait 1) and A, k, AFC, WFC and ABW (trait 2) of females of the Canchim breed

\begin{tabular}{|c|c|c|c|c|c|c|}
\hline \multirow[t]{2}{*}{$\begin{array}{l}\text { Caract. } 2^{1} \\
\text { Trait } 1,2^{* *}\end{array}$} & \multicolumn{2}{|c|}{$\begin{array}{l}\mathrm{P} 18(\text { caract. } 1)^{1} \\
B W 18(\text { trait } 1)^{1}\end{array}$} & \multicolumn{2}{|c|}{$\begin{array}{l}\text { Caract. } 2^{*} \\
\text { Trait } 2^{*} \\
\end{array}$} & \multicolumn{2}{|c|}{$\begin{array}{c}\text { Caract. } 1,2^{* *} \\
\text { Trait } 1,2^{* *} \\
\end{array}$} \\
\hline & $\sigma^{2}{ }_{a 1}$ & $\sigma^{2}{ }_{\mathrm{e} 1}$ & $\sigma^{2}{ }_{a 2}$ & $\sigma_{\mathrm{e} 2}^{2}$ & $\sigma_{\mathrm{a} 1 \mathrm{a} 2}$ & $\sigma_{\mathrm{e} 1 \mathrm{e} 2}$ \\
\hline $\mathrm{A} / A$ & 412 & 767 & 3888 & 5719 & 261 & 92 \\
\hline $\mathrm{K} / k$ & 419 & 764 & 5 & 14 & 58 & 89 \\
\hline $\mathrm{IPP} / A F C$ & 425 & 759 & 3820 & 26511 & -367 & -988 \\
\hline $\mathrm{PPP} / W F C$ & 409 & 773 & 987 & 1649 & 411 & 166 \\
\hline $\mathrm{PAD} / A B W$ & 422 & 762 & 1109 & 1806 & 409 & 451 \\
\hline
\end{tabular}

${ }^{1} \mathrm{P} 18, \mathrm{~A}, \mathrm{k}$, IPP, PPP e PAD = peso $(\mathrm{kg})$ aos 18 meses de idade, estimativas dos parâmetros $A(\mathrm{~kg})$ e k (mês $\left.{ }^{-1}\right)$ da curva de crescimento, idade (dias) e peso $(\mathrm{kg})$ ao primeiro parto e peso $(\mathrm{kg})$ à idade adulta, respectivamente.

$2 \sigma^{2}$ a $\sigma^{2}$ e $\sigma_{\mathrm{a} 1 \mathrm{a} 2}$ e $\sigma_{\mathrm{e} 1 \mathrm{e} 2}=$ componentes de variância genético aditivo direto residual e de covariância aditiva direta e residual, respectivamente.

*variâncias de k x 100.000; ${ }^{* *}$ covariâncias com k x 10.000

${ }^{1} B W 18, A, k, A F C, W F C$ and $A B W=$ body weight $(\mathrm{kg})$ at 18 months of age, estimates of the parameters $A(k g)$ and $k(m o n t h-1)$ of the growth curve, age (days) and weight $(\mathrm{kg})$ at first calving, and adult weight $(\mathrm{kg})$, respectively.

$2 \sigma^{2}{ }_{a}, \sigma^{2}{ }_{e}, \sigma_{a 1 a 2}$ and $\sigma_{e 1 e 2}=$ genetic additive direct and residual variance components, and additive and residual covariance components.

* variances of $k \times 100.000 ;{ }^{* *}$ covariances with $k \times 10.000$ 
há pouca variação genética aditiva na característica, sugerindo baixo progresso genético pela seleção.

As estimativas das correlações genéticas, fenotípicas e residuais de PD, P12 e P18 com as estimativas dos parâmetros A e k da curva de crescimento, IPP, PPP e PAD são apresentadas na Tabela 4. As estimativas da correlação genética entre os efeitos diretos e maternos para PD variaram de $-0,46$ a $-0,56$ (Tabela 4), com média de $-0,51$, concordando com o valor de - 0,54 obtido por Alencar et al. (1998) para o mesmo rebanho, porém maiores que o valor de 0,01 obtido por Mello et al. (2002), para o mesmo conjunto de dados, mas por análise tricaráter dos pesos ao nascimento, à desmama e aos 12 meses de idade. Os resultados obtidos indicam que a seleção para melhorar os efeitos aditivos diretos de PD deve resultar em piora nos efeitos aditivos maternos ou vice versa, e que a seleção para qualquer um deles não deve ser tão efetiva.

As correlações genéticas de PD $(0,46), \mathrm{P} 12$ $(0,39)$ e P18 $(0,21)$ com a estimativa do parâmetro A da curva de crescimento foram positivas e reduziram com o aumento da idade do animal (Tabela 4). Jenkins et al. (1991) encontraram o valor de 0,60 para a correlação genética entre P12 e o parâmetro A (pelo modelo de Brody) em fêmeas bovinas de corte. Oliveira (1995), no Brasil, trabalhando com o modelo de Von Bertalanffy, obteve correlação de 0,89 , para fêmeas da raça Guzerá, enquanto Rosa et al. (1979), usando o modelo de Brody, estimaram correlação bem mais baixa $(0,09)$, para animais Nelore. Já Silva et al. (2000) obtiveram o valor de 0,19 para a correlação genética de $\mathrm{P} 12$ de machos com o parâmetro A de fêmeas, no mesmo rebanho deste estudo. Os resultados obtidos neste trabalho sugerem que a seleção para peso, principalmente até os 12 meses de idade, deve resultar em aumento no peso à maturidade das fêmeas.

As correlações genéticas de PD $(0,02), \mathrm{P} 12$ $(0,31)$ e P18 $(0,42)$ com o parâmetro $\mathrm{k}$ da curva de crescimento foram positivas e aumentaram com o aumento da idade do animal (Tabela 4), discordando de Brown et al. (1972), que obtiveram o valor de - 0,23 para P12 e k, mas utilizando o modelo de Brody para descrever a curva de crescimento de fêmeas da raça Hereford. Com o modelo de Von Bertalanffy, Oliveira (1995) estimou o valor de $-0,70$ para a correlação genética de P12 e k em um rebanho Guzerá. Por outro lado, ainda para P12 e k, Brown et al. (1972) e Jenkins et al. (1991) reportaram valores positivos iguais a 0,95 e 0,36, para fêmeas Angus e de várias raças, respectivamente. Os valores das correlações genéticas de P12 e P18 com o parâmetro k, obtidas neste trabalho, indicam que a seleção para peso nessas idades deve resultar em maior taxa de maturação das fêmeas. Este resultado parece contraditório considerando-se a correlação genética negativa $(-0,74)$ entre as estimativas dos parâmetros da curva de crescimento, obtida neste trabalho, e as correlações positivas obtidas para os pesos e o parâmetro A. Entretanto, observa-se que, além de a correlação genética entre os parâmetros $\mathrm{A}$ e k não ser igual a um $(1,0)$, a correlação genética dos pesos com os parâmetros da curva tende a diminuir de 0,46 a 0,21 com o aumento da idade, para o parâmetro A e aumentar de valor próximo de zero a 0,42 , para o parâmetro $\mathrm{k}$, mostrando relação dos valores das correlações dos pesos com os parâmetros A e k alta/ baixa (PD), média/média (P12) e baixa/alta (P18). Desta maneira, nas fêmeas da raça Canchim do rebanho estudado, a seleção para maior PD resultará em maior peso assintótico sem afetar a taxa de maturação, a seleção para maior $\mathrm{P} 12$ resultará em maiores A e k, e a seleção para maior P18 resultará em maior $\mathrm{k}$ com pouco aumento de A.

As correlações genéticas dos pesos da desmama até os 18 meses de idade com a idade ao primeiro parto foram negativas e de magnitude baixa a média $(-0,12$ a $-0,32$; Tabela 4). Estes resultados discordam daqueles obtidos por Mariante (1978) que verificou, pelo método dos quadrados mínimos, correlações genéticas positivas e decrescentes $(0,65$ a 0,12$)$ dos pesos do nascimento aos 24 meses de idade com a idade ao primeiro parto de fêmeas da raça Nelore. Barbosa (1991) obteve, para o mesmo rebanho deste estudo e pelo método dos quadrados mínimos, correlação genética igual a 0,37 para o peso à desmama e a idade ao primeiro parto. Por outro lado, este mesmo autor relatou correlações genéticas negativas e decrescentes $(-0,64$ a $-0,23)$ dos pesos aos 12 , aos 18 e aos 24 meses de idade com a idade ao primeiro parto. Silva et al. (2000), para o mesmo conjunto de dados e metodologia deste trabalho, obtiveram o valor de $-0,58$ para a correlação genética do peso de machos aos 12 meses de idade e a idade ao primeiro parto das fêmeas. Os resultados obtidos neste estudo indicam que a seleção de fêmeas com base nos pesos deve resultar em redução na idade ao primeiro parto. Estes resultados eram esperados, uma vez que o desenvolvimento ponderal das fêmeas foi um dos critérios

\footnotetext{
R. Bras. Zootec., v.32, n.4, p.880-886, 2003
} 
utilizados para a entrada das novilhas em reprodução.

As correlações genéticas entre os pesos nas várias idades e o peso ao primeiro parto foram positivas e altas ( 0,65 a 0,77 ; Tabela 4 ), concordando com o valor de 0,69 obtido por Silva et al. (2000), para o peso de machos aos 12 meses de idade e o peso das fêmeas ao primeiro parto. Estes resultados indicam que a seleção para aumentar o peso das fêmeas da desmama aos 18 meses de idade aumentará também o peso ao primeiro parto.

Os valores obtidos para as correlações genéticas entre os pesos da desmama aos 18 meses de idade e o peso adulto das fêmeas foram de magnitude média a alta (0,42 a 0,66; Tabela 4), sugerindo que a seleção para peso em idades jovens deve aumentar o peso adulto das fêmeas. Esses resultados concordam com aqueles obtidos por Barbosa (1991), na raça Canchim, e por Bullock et al. (1993), na raça Hereford, que verificaram correlação genética alta e positiva entre os pesos em idades jovens e o peso adulto das fêmeas.

As correlações fenotípicas e residuais de PD, P12 e P18 com as outras características têm magnitude baixa a média e, geralmente, apresentam o mesmo sinal da correlação genética correspondente (Tabela 4). Com PPP e PAD, as correlações foram positivas e indicam, principalmente para $\mathrm{PAD}$, que parte dos fatores genéticos e não genéticos que influenciam o desenvolvimento das fêmeas da desmama até os 18 meses de idade também influencia os pesos ao primeiro parto e à idade adulta. Barbosa
(1991), na raça Canchim, obteve correlações fenotípicas e ambientais dos pesos do nascimento aos 24 meses de idade com o peso adulto de fêmeas que variaram, respectivamente, de 0,33 a 0,49 e de 0,10 a 0,31 . Bullock et al. (1993) estimaram o valor de 0,42 para a correlação fenotípica entre os pesos aos 12 meses de idade e à maturidade de fêmeas da raça Hereford.

As correlações fenotípicas e residuais com o parâmetro $\mathrm{A}$ são baixas e, às vezes, negativas (Tabela 4). As correlações com o parâmetro k são baixas para PD e médias e positivas para P12 e P18, sugerindo que os fatores que contribuem para maior peso também contribuem para maior taxa de maturação. Utilizando o modelo de Brody para descrever o crescimento de fêmeas de corte, Lopez de Torre \& Rankin (1978) obtiveram os valores de 0,33 e 0,32 para a correlação fenotípica entre o parâmetro A e o peso aos 14 meses de idade para as raças Angus e Hereford, respectivamente. Jenkins et al. (1991), utilizando mesmo modelo, relatam os valores de 0,39 e 0,43 para as correlações fenotípicas de P12 com os parâmetros A e k, respectivamente. No Brasil, Rosa et al. (1979), também para o modelo Brody, estimaram a correlação fenotípica de $-0,03$ entre P12 e o parâmetro A, na raça Nelore, enquanto que Oliveira (1995) obteve os valores de 0,37 e 0,28 de P12 com os parâmetros A e k, respectivamente, na raça Guzerá.

As correlações fenotípicas e residuais com IPP

Tabela 4 - Estimativas ${ }^{2}$ de correlações genéticas, fenotípicas e residuais de A, k, IPP, PPP e PAD (caract.2) com PD, P12 e P18 (caract.1) de fêmeas da raça Canchim

Table 4 - Estimates ${ }^{2}$ of genetic, phenotypic and residual correlations of BWW, BW12 and BW18 (trait 1) with A, k, AFC, WFC and ABW of females of the Canchim breed

\begin{tabular}{|c|c|c|c|c|c|c|c|c|c|c|}
\hline \multirow{3}{*}{$\begin{array}{l}\text { Caract. } 2^{1} \\
\text { Trait } 2^{1}\end{array}$} & \multicolumn{10}{|c|}{$\begin{array}{c}\text { Característica } 1^{1} \\
\text { Trait } 1^{1}\end{array}$} \\
\hline & \multicolumn{4}{|c|}{$P D(\mathrm{BWW})$} & \multicolumn{3}{|c|}{$P 12(\mathrm{BW} 12)$} & \multicolumn{3}{|c|}{ P18(BW18) } \\
\hline & $\mathrm{r}_{\mathrm{a} 1 \mathrm{a} 2}$ & $\mathrm{r}_{\mathrm{a} 1 \mathrm{~m} 1}$ & $r_{p 1 p 2}$ & $\mathrm{r}_{\mathrm{e} 1 \mathrm{e} 2}$ & $\mathrm{r}_{\mathrm{a} 1 \mathrm{a} 2}$ & $r_{p 1 p 2}$ & $\mathrm{r}_{\mathrm{e} 1 \mathrm{e} 2}$ & $\mathrm{r}_{\mathrm{a} 1 \mathrm{a} 2}$ & $\mathrm{r}_{\mathrm{p} 1 \mathrm{p} 2}$ & $\mathrm{r}_{\mathrm{e} 1 \mathrm{e} 2}$ \\
\hline $\mathrm{A} / \mathrm{A}$ & 0,46 & $-0,52$ & 0,10 & $-0,10$ & 0,39 & $-0,05$ & $-0,28$ & 0,21 & 0,11 & 0,04 \\
\hline $\mathrm{k} / \mathrm{k}$ & 0,02 & $-0,56$ & 0,13 & 0,18 & 0,31 & 0,45 & 0,53 & 0,42 & 0,32 & 0,27 \\
\hline IPP/AFC & $-0,12$ & $-0,55$ & $-0,11$ & $-0,13$ & $-0,32$ & $-0,20$ & $-0,18$ & $-0,29$ & $-0,23$ & $-0,22$ \\
\hline PPP/WFC & 0,66 & $-0,46$ & 0,31 & 0,09 & 0,77 & 0,37 & 0,14 & 0,65 & 0,33 & 0,15 \\
\hline PAD/ABW & 0,42 & $-0,48$ & 0,37 & 0,30 & 0,66 & 0,42 & 0,29 & 0,60 & 0,46 & 0,38 \\
\hline \multicolumn{11}{|c|}{ 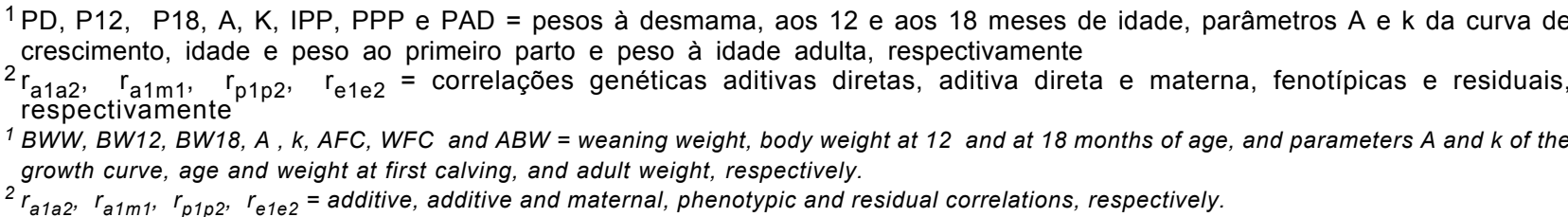 } \\
\hline
\end{tabular}

R. Bras. Zootec., v.32, n.4, p.880-886, 2003 
são baixas e negativas (Tabela 4), indicando que os fatores que contribuem para maior peso não prejudicam a idade ao primeiro parto das novilhas. Mariante (1978) e Barbosa (1991) observaram, nas raças Nelore e Canchim, correlações fenotípicas e ambientais dos pesos da desmama aos 24 meses de idade com a idade ao primeiro parto com valores negativos, e Alencar \& Bügner (1986) também observaram associação favorável entre peso aos 24 meses de idade e idade ao primeiro parto de novilhas da raça Canchim.

\section{Conclusões}

A seleção para maior peso à desmama, aos $12 \mathrm{e}$ aos 18 meses de idade nas fêmeas da raça Canchim do rebanho estudado deve resultar em progresso genético nessas características e em maior taxa de maturação e maior precocidade reprodutiva (menor idade ao primeiro parto), mas com aumentos nos seus pesos adulto e ao primeiro parto. A utilização do peso à desmama, aos 12 e aos 18 meses de idade como critério de seleção para fềmeas deve ser acompanhada de monitoramento constante do peso adulto das vacas.

\section{Literatura Citada}

ALENCAR, M.M.; BUGNER, M. Estudo de idade ao primeiro parto de vacas da raça Canchim. Revista da Sociedade Brasileira de Zootecnia, v.15, n.2, p.151-156, 1986.

ALENCAR, M.M.; SILVA, A.H.G.; BARBOSA, P.F. Efeitos da consagüinidade sobre os pesos ao nascimento e à desmama de bezerros da raça Canchim. Revista da Sociedade Brasileira de Zootecnia, v.10, n.1, p.151-156, 1981.

ALENCAR, M.M.; TREMATORE, R.L.; BARBOSA, P.F. et al. Efeitos da linhagem citoplasmática sobre características de crescimento em bovinos da raça Canchim. Revista Brasileira de Zootecnia, v.27, n.2, p.272-276, 1998.

BARBOSA, P.F. Análise genético-quantitativa de características de crescimento e reprodução em fêmeas da raça Canchim. Ribeirão Preto: Universidade de São Paulo, 1991. 237p. Tese (Doutorado em Genética) - Universidade de São Paulo, 1991.

BERTALANFFY, L.V. Quantitative laws in metabolism and growth. The Quaterly Review of Biology, v.32, p.217-230, 1957.

BOLDMAN, K.; KRIESE, L.; Van VLECK, L.D. et al. A manual for use of MTDFREML - A set of programs to obtain estimates of variances and covariances. United States Department of Agriculture-Agricultural Research Service, 1993. 120p.

BROWN, J.E.; BROWN, C.J.; BUTTS, W.T. A discussion of the genetic aspects of weight, mature weight and rate of maturing in Hereford and Angus cattle. Journal of Animal Science, v.34, n.4, p.525-537, 1972.

BULLOCK, K.D.; BERTRAND, J.K.; BENYSHEK, L.L. Genetic and environmental parameters for mature weight and other growth measures in Polled Hereford cattle. Journal of Animal Science, v.71, n.7, p.1737-1741, 1993.

DeNISE, R.S.K.; BRINKS, J.S.; RICHARDSON, G.V. et al. Relationships among the growth curve parameters and selected productivity traits in beef cows. Journal of Animal Science, v.57, n.1, p.149, 1983. (Supplement)

FREITAS, A.R.; ALENCAR, M.M.; SILVA, A.S. Ajuste de modelos não lineares em bovinos de corte. I. Padrão de crescimento da população. In: REUNIÃO ANUAL DA SOCIEDADE BRASILEIRA DE ZOOTECNIA, 35., 1998, Botucatu. Anais... Botucatu: Sociedade Brasileira de Zootecnia, p.341-343. 1998.

JENKINS, T.G.; KASPS, M.; CUNDIF, L.V. et al. Evaluations of between and within breed variation in measures of weightage relationships. Journal of Animal Science, v.69, n.8, p.3118-3128, 1991.

LOPEZ DE TORRE, G.; RANKIN, B.J. Factors affecting growth curve parameters of Hereford and Brangus cows. Journal of Animal Science, v.46, n.3, p.604-617, 1978.

MARIANTE, A.S. Growth and reproduction in Nelore cattle in Brazil: genetic parameters and effects of environmental factors. Gainesville: University of Florida, 1978. 131 p. Thesis (Phylosophy Doctor) - University of Florida, 1978.

MELLO, S.P.; ALENCAR, M.M.; SILVA, L.O.C. et al. Estimativas de co(variâncias) e tendências genéticas para pesos em um rebanho Canchim. Revista Brasileira de Zootecnia, v.31, n.3, p.1707-1714, 2002.

OLIVEIRA, H.N. Análise genético-quantitativa da curva de crescimento de fêmeas da raça Guzerá. Ribeirão Preto: Universidade de São Paulo, 1995. 73p. Tese (Doutorado em Genética) - Universidade de São Paulo, 1995.

ROSA, A.N.; SILVA, M.A.; SILVA, J.C. et al. Análise genética de peso à maturidade e do grau de maturidade de animais da raça Nelore. Revista da Sociedade Brasileira de Zootecnia, v.8, n.1, p.43-56, 1979.

SILVA, A.M. Parâmetros genéticos para peso e perímetro escrotal de machos e características reprodutivas e de crescimento de fêmeas, na raça Canchim. Jaboticabal: Universidade Estadual Paulista, 1999. 88p. Dissertação (Mestrado em Zootecnia) - Universidade Estadual Paulista, 1999.

SILVA, A.M.; ALENCAR, M.M.; FREITAS, A.R. et al. Herdabilidade e correlações genéticas para peso e perímetro escrotal de machos e características reprodutivas e de crescimento de fêmeas, na raça Canchim. Revista Brasileira de Zootecnia, v.29, n.6, p.2223-2230, 2000. (Suplemento 2)

STATISTICAL ANALYSIS SYSTEMS. Statistical analysis systems user's guide. 4.ed. Cary: 1996. v.2.

Recebido em: 13/07/01 Aceito em: 05/04/02 